\title{
Propagating fronts in reaction-transport systems with memory
}

DOI:

10.1016/j.physleta.2007.06.044

\section{Document Version}

Final published version

Link to publication record in Manchester Research Explorer

\section{Citation for published version (APA):}

Yadav, A., Fedotov, S., Méndez, V., \& Horsthemke, W. (2007). Propagating fronts in reaction-transport systems with memory. Physics Letters, Section A: General, Atomic and Solid State Physics, 371(5-6), 374-378.

https://doi.org/10.1016/j.physleta.2007.06.044

\section{Published in:}

Physics Letters, Section A: General, Atomic and Solid State Physics

\section{Citing this paper}

Please note that where the full-text provided on Manchester Research Explorer is the Author Accepted Manuscript or Proof version this may differ from the final Published version. If citing, it is advised that you check and use the publisher's definitive version.

\section{General rights}

Copyright and moral rights for the publications made accessible in the Research Explorer are retained by the authors and/or other copyright owners and it is a condition of accessing publications that users recognise and abide by the legal requirements associated with these rights.

\section{Takedown policy}

If you believe that this document breaches copyright please refer to the University of Manchester's Takedown Procedures [http://man.ac.uk/04Y6Bo] or contact uml.scholarlycommunications@manchester.ac.uk providing relevant details, so we can investigate your claim.

\section{OPEN ACCESS}




\title{
Propagating fronts in reaction-transport systems with memory
}

\author{
A. Yadav ${ }^{\mathrm{a}, 1}$, Sergei Fedotov ${ }^{\mathrm{b}}$, Vicenç Méndez ${ }^{\mathrm{c}}$, Werner Horsthemke ${ }^{\mathrm{a}, *}$ \\ a Department of Chemistry, Southern Methodist University, Dallas, TX 75275-0314, USA \\ $\mathrm{b}$ School of Mathematics, The University of Manchester, Manchester M60 1DQ, UK \\ ${ }^{\mathrm{c}}$ Grup de Física Estadística, Departament de Física, Universitat Autònoma de Barcelona, E-08193 Bellaterra, Spain
}

Received 23 February 2007; received in revised form 5 June 2007; accepted 12 June 2007

Available online 23 June 2007

Communicated by C.R. Doering

\begin{abstract}
In reaction-transport systems with non-standard diffusion, the memory of the transport causes a coupling of reactions and transport. We investigate the effect of this coupling for systems with Fisher-type kinetics and obtain a general analytical expression for the front speed. We apply our results to the specific case of subdiffusion.
\end{abstract}

(C) 2007 Elsevier B.V. All rights reserved.

PACS: 82.40.Ck; 05.40.Fb; 87.23.Cc; 05.60.-k

Keywords: Memory kernel; Reaction-transport system; Subdiffusion; Propagating front

\section{Introduction}

Propagating fronts are nonequilibrium phenomena that occur in many areas of physics, chemistry, biology, ecology, and other fields [1-3]. A particularly well-studied class of applications consists of reaction-diffusion systems, where the combination of reaction processes and dispersal through diffusion is able to support a shape preserving and uniformly translating solution under certain well-understood conditions $[2,4]$.

Recently, emphasis has focused on understanding front propagation in complex systems where the dispersal of particles differs from Brownian motion or standard diffusion. To remedy the unphysical nature of Fickian diffusion, namely transport with infinite speed, hyperbolic evolution equations, such as reaction random walks [5], time-delayed hyperbolic reactiondiffusion equations [6-8], and reaction-telegraph equations $[9,10]$, have been introduced and analyzed in the context of

\footnotetext{
* Corresponding author. Fax: +1 2147684089.

E-mail addresses: ayadav1@1su.edu (A. Yadav),

sergei.fedotov@manchester.ac.uk (S. Fedotov), vicenc.mendez@uab.es

(V. Méndez), whorsthe@ smu.edu (W. Horsthemke).

1 Current address: Department of Physics and Astronomy, Louisiana State University, Baton Rouge, LA 70803-4001, USA.
}

front propagation. Front propagation in systems where the dispersal is through anomalous diffusion has also been the subject of recent investigations [11-15].

An approach employed in several studies consists of replacing the standard Fickian relation between the flux, $J(x, t)$, and the gradient of the concentration for a species, $\rho(x, t)$,

$J(x, t)=-D \partial_{x} \rho(x, t)$,

by a more general form [16],

$J(x, t)=-\int_{0}^{t} \theta\left(t-t^{\prime}\right) \partial_{x} \rho\left(x, t^{\prime}\right) d t^{\prime}$,

through the introduction of the memory kernel $\theta(t)$. Invoking the continuity equation for the concentration,

$\partial_{t} \rho(x, t)+\partial_{x} J(x, t)=f(\rho(x, t))$,

one arrives at a generalization of the standard reaction-diffusion equation:

$\partial_{t} \rho(x, t)=\int_{0}^{t} \theta\left(t-t^{\prime}\right) \partial_{x x} \rho\left(x, t^{\prime}\right) d t^{\prime}+f(\rho(x, t))$. 
The reaction term $f(\rho)$ plays the role of a source in the continuity condition (3). A delta function form for the memory kernel results in the recovery of the standard reaction-diffusion equation, while exponential decay and fractional derivative kernels lead to a hyperbolic reaction-diffusion equation and to a fractional reaction-diffusion equation, respectively.

In both the standard reaction-diffusion picture, and its generalization, Eq. (4), the effects of reactions and dispersal are separable and combine additively to influence the total spatiotemporal evolution of the concentration field of a given species. However, in a recent work [17] we have shown that this is not true for non-standard diffusive transport. Memory effects associated with such transport lead to the coupling of transport and reactions. The main goal of this Letter is to analyze the influence of the coupling between reactions and dispersal on front propagation.

\section{Generalized reaction-transport equations}

In this section we briefly recapitulate the results of Ref. [17], based upon which we write down a generalized Fick's first law taking into account the coupling between reaction processes and dispersal. In Ref. [17] we employed as our starting point a mesoscopic approach, namely the formalism proposed by Vlad and Ross based on continuous time random walks (CTRWs) with reactions [18]. In this formalism, the concentration fields of the various species obey a set of nonlinear, age-dependent evolution equations that account for the transport and transformation processes in the system. The long-time and large-spatial scale limit ("diffusive limit") of those equations results in the following generalized reaction-diffusion equations governing the evolution of a system with reactions and spatial dispersal, see Eq. (27) in Ref. [17],

$$
\begin{aligned}
\frac{\partial \rho_{i}(x, t)}{\partial t}= & R_{i}^{+}(\rho(x, t))-R_{i}^{-}(\rho(x, t)) \\
& +\sigma_{i}^{2} \partial_{x x}\left\{\int_{0}^{t} \Theta_{i}\left(t-t^{\prime}\right) \rho_{i}\left(x, t^{\prime}\right)\right. \\
& \left.\times \exp \left[-\int_{t^{\prime}}^{t} \frac{R_{i}^{-}\left(\rho\left(x, t^{\prime \prime}\right)\right)}{\rho_{i}\left(x, t^{\prime \prime}\right)} d t^{\prime \prime}\right] d t^{\prime}\right\} .
\end{aligned}
$$

Here, $\rho_{i}(x, t)$ is the concentration field of species $i$, and the species undergo reactions, birth-and-death processes, with a birth rate $R_{i}^{+}(\rho(x, t)) \geqslant 0$ and a death rate $R_{i}^{-}(\rho(x, t)) \geqslant 0$, where $\rho(x, t)=\left(\rho_{1}(x, t), \rho_{2}(x, t), \ldots, \rho_{n}(x, t)\right)$. The total reaction terms are defined as $f_{i}(\rho(x, t)) \equiv R_{i}^{+}(\rho(x, t))-$ $R_{i}^{-}(\rho(x, t))$. The operator $\sigma_{i}^{2} \partial_{x x}$ has its origins in the underlying spatial jump probability distribution, $\lambda_{i}(x)$, which has the form $\lambda_{i}(k)=1-\sigma_{i}^{2} k^{2}$ in conjugate Fourier space. The kernel $\Theta_{i}(t)$ is related to the waiting time probability distribution function $(\mathrm{PDF}), \phi_{i}(t)$, of the underlying CTRW through

$$
\hat{\Theta}_{i}(u) \equiv \frac{u \hat{\phi}_{i}(u)}{1-\hat{\phi}_{i}(u)}
$$

in conjugate Laplace space, where the Laplace-transformed function is denoted by a hat. The presence of both the kernel $\Theta_{i}\left(t-t^{\prime}\right)$, related to the waiting time PDF of the CTRW, and the death rate $R_{i}^{-}(\rho(x, t))$ in the memory term in Eq. (5) indicates that the effects of reaction and non-standard diffusive transport are not separable, in contrast to the standard reaction-diffusion equation or its generalization (4). It is worth noting that Eq. (5) simplifies to a standard reaction-diffusion equation if the CTRW is Markovian, i.e., the waiting times are exponentially distributed, $\phi_{i}(t)=\left(1 / \eta_{i}\right) e^{-t / \eta_{i}}$. In this case $\hat{\Theta}_{i}(u)=1 / \eta_{i}$, and therefore, $\Theta_{i}(t)=\delta(t) / \eta_{i}$.

The Vlad-Ross formalism and the generalized reactiondiffusion equation (5) are mean-field equations, i.e., they represent a deterministic, continuum description of a system that is discrete at the microscopic level, and are valid in the thermodynamic limit as the number of particles goes to infinity. Systems with a finite number of particles display internal fluctuations. For standard reaction-diffusion systems, the effect of these fluctuations on the dynamics of propagating fronts has been studied numerically and analytically [19-23].

The generalized reaction-diffusion equation (5) suggests that one can write down a generalized Fick's first law that takes into account the coupling between reactions and dispersal,

$$
\begin{aligned}
J(x, t)= & -\sigma^{2} \partial_{x} \int_{0}^{t} \Theta\left(t-t^{\prime}\right) \\
& \times \exp \left[-\int_{t^{\prime}}^{t} \frac{R^{-}\left(\rho\left(x, t^{\prime \prime}\right)\right)}{\rho\left(x, t^{\prime \prime}\right)} d t^{\prime \prime}\right] \rho\left(x, t^{\prime}\right) d t^{\prime} .
\end{aligned}
$$

Invoking the continuity equation (3) and substituting Eq. (7) into it, we recover Eq. (5). For a general transport process with memory, the flux at the present time does not instantaneously follow the gradient in concentration, nor does it follow the gradient in concentration at previous times, but rather the gradient in concentration at previous times weighted by the specific death rate.

\section{Front propagation in generalized reaction-transport equations}

Having shown that the proper generalization of a reactiondiffusion equation for non-standard diffusive transport couples the reaction and transport processes, we seek to analyze the influence of this coupling on front propagation. For simplicity we examine Eq. (5) for a single species $i=1$ and drop the suffix 1 . Moreover, the nonlinear kinetic term $f(\rho(x, t))$ is assumed to be of the Fisher-Kolmogorov-Petrovskii-Piskunov (FKPP) type [24], i.e., $\max _{\rho \in(0,1)} f(\rho) / \rho=U, f(0)=0$, and $f(1)=0$, where $U$ is the constant growth rate. We will in particular address the case of subdiffusive transport.

The effect of subdiffusion on front propagation has been investigated previously, using CTRW-based formalisms other than the Vlad-Ross formalism [11,13-15]. Those approaches are valid as long as the kinetic term can be interpreted as a source term, i.e., as the rate with which new particles are cre- 
ated. This requires that $f(\rho) \geqslant 0$ for the range of densities considered. If the kinetic term results from separate birth and death processes, the situation usually encountered in chemical systems, then, as we will show below, the predictions of those approaches remain valid, as long as the death rate depends nonlinearly on the density.

The specific chemical scheme we consider here is the branching-coalescence scheme,

$\mathrm{B}+\mathrm{X} \longrightarrow \mathrm{X}+\mathrm{X}$

$\mathrm{X}+\mathrm{X} \longrightarrow \mathrm{B}+\mathrm{X}$,

where the species B is a pool chemical, i.e., its concentration is kept constant. The suitably nondimensionalized mass-action rate term of this scheme is given by the logistic kinetic term

$f(\rho)=U \rho(1-\rho)=R^{+}(\rho)-R^{-}(\rho)$,

which is the most commonly used form of FKPP-type kinetics. For the branching-coalescence scheme (8), the birth rate is $R^{+}(\rho)=U \rho$, and the death rate is $R^{-}(\rho)=U \rho^{2}$. The evolution equation for this kinetic scheme is

$$
\begin{aligned}
\frac{\partial \rho(x, t)}{\partial t}= & U \rho(x, t)-U[\rho(x, t)]^{2} \\
& +\sigma^{2} \partial_{x x}\left\{\int_{0}^{t} \Theta\left(t-t^{\prime}\right) \rho\left(x, t^{\prime}\right)\right. \\
& \left.\times \exp \left[-\int_{t^{\prime}}^{t} U \rho\left(x, t^{\prime \prime}\right) d t^{\prime \prime}\right] d t^{\prime}\right\} .
\end{aligned}
$$

We choose "natural" initial conditions, i.e., initial conditions that are localized or that decay faster than exponentially, e.g., Heaviside initial conditions, $\rho(x, 0)=1$ for $x \leqslant 0$ and $\rho(x, 0)=0$ for $x>0$. Our choice of initial conditions ensures that the propagation speed of such a front is minimal. We employ the Hamilton-Jacobi approach [11] to derive the speed of propagating fronts exhibited by Eq. (10). This approach relies on a hyperbolic scaling $x \rightarrow x / \epsilon$ and $t \rightarrow t / \epsilon$, with the rescaled concentration, $\rho^{\epsilon}(x, t)=\rho(x / \epsilon, t / \epsilon)$ written in terms of an action functional $G^{\epsilon}$ as $\rho^{\epsilon}(x, t)=\exp \left[-G^{\epsilon}(x, t) / \epsilon\right]$. In terms of $G^{\epsilon}$, Eq. (10) takes the form,

$$
\begin{aligned}
-\partial_{t} G^{\epsilon}= & \sigma^{2} \int_{0}^{t / \epsilon} \Theta\left(t^{\prime}\right)\left\{e^{-\left[G^{\epsilon}\left(x, t-\epsilon t^{\prime}\right)-G^{\epsilon}(x, t)\right] / \epsilon}\right. \\
& \left.\times\left[\partial_{x} G^{\epsilon}\left(x, t-\epsilon t^{\prime}\right)\right]^{2}+O(\epsilon)\right\} \\
& \times \exp \left[-\int_{t / \epsilon-t^{\prime}}^{t / \epsilon} U e^{-G^{\epsilon} / \epsilon} d t^{\prime \prime}\right] d t^{\prime} \\
& +f\left(e^{-G^{\epsilon} / \epsilon}\right) / e^{-G^{\epsilon} / \epsilon} .
\end{aligned}
$$

The equation for the evolution of $G(x, t)$ is obtained by taking the limit $\epsilon \rightarrow 0$ [15], resulting in

$-\partial_{t} G=\left(\partial_{x} G\right)^{2} \sigma^{2} \int_{0}^{\infty} \Theta\left(t^{\prime}\right) e^{t^{\prime} \partial_{t} G} d t^{\prime}+U$.
Identifying a Hamiltonian $H=-\partial_{t} G$ and a generalized momentum $p=-\partial_{x} G$, one can rewrite Eq. (12) as

$H=p^{2} \sigma^{2} \hat{\Theta}(H)+U$,

where $\hat{\Theta}(H)$ is the Laplace transform of $\Theta(t)$, and the Hamiltonian is the conjugate variable in Laplace space. After solving Hamilton's equations, and realizing that at the front position $G(x, t)=0$, we find that the front speed is given by [15]

$v(H)=H \sqrt{\frac{\sigma^{2} \hat{\Theta}(H)}{H-U}}, \quad \frac{\partial p}{\partial H}=\frac{p}{H}$.

It is interesting to note that performing the hyperbolic scaling analysis of Eq. (4) with logistic kinetics [15] leads to the same evolution equation for $G(x, t)$, namely Eq. (12). Therefore, Eq. (4) with no coupling between reaction and dispersal, and Eq. (10) with the coupling present, exhibit fronts with the same speed of propagation, leading to the conclusion that the coupling has no effect on the speed of front propagation. This conclusion, however, is only true for certain forms of the reaction term $f(\rho)$, namely if the death rate $R^{-}(\rho)$ is nonlinear.

To show this, we consider a generalization of the branchingcoalescence scheme (8), namely the first Schlögl model [25]:

$\mathrm{B}+\mathrm{X} \longrightarrow \mathrm{X}+\mathrm{X}$,
$\mathrm{X}+\mathrm{X} \longrightarrow \mathrm{B}+\mathrm{X}$,
$\mathrm{A}+\mathrm{X} \longrightarrow \mathrm{C}$,

where $\mathrm{A}$ and $\mathrm{C}$ are also pool chemicals. An appropriately scaled version of this scheme leads to a rate term of the form $f(\rho)=$ $b \rho-a \rho-(b-a) \rho^{2}(b>a>0)$, with the birth rate $R^{+}=$ $b \rho$ and the death rate $R^{-}=a \rho+(b-a) \rho^{2}$. Clearly, $\rho=0$ and $\rho=1$ are steady states for this model, as is the case for the branching-coalescence model. However, the Schlögl model contains separate linear birth and death terms, which results in the evolution equation

$$
\begin{aligned}
\frac{\partial \rho(x, t)}{\partial t}= & (b-a) \rho(x, t)-(b-a)[\rho(x, t)]^{2} \\
& +\sigma^{2} \partial_{x x}\left\{\int_{0}^{t} \Theta\left(t-t^{\prime}\right) \rho\left(x, t^{\prime}\right)\right. \\
& \left.\times \exp \left[-\int_{t^{\prime}}^{t}\left\{(b-a) \rho\left(x, t^{\prime \prime}\right)+a\right\} d t^{\prime \prime}\right] d t^{\prime}\right\} .
\end{aligned}
$$

For standard diffusion, i.e., a delta function form of the kernel $\Theta(t)$, and identifying $U=b-a$ in Eq. (16), we recover the usual reaction-diffusion equation with a logistic growth term. Here the linear birth term and the linear death term combine to provide an effective linear growth term $U \rho$. However, for nonstandard diffusive transport, the linear growth term and the linear death term play different roles in the way they influence the evolution of the concentration $\rho(x, t)$. The coupling between reactions and dispersal for non-standard diffusive transport is mediated by the combined death term with $a \rho$ as the linear component. 
Performing a hyperbolic scaling analysis of Eq. (16), we obtain the governing equation for $G(x, t)$;

$-\partial_{t} G=\left(\partial_{x} G\right)^{2} \sigma^{2} \int_{0}^{\infty} \Theta\left(t^{\prime}\right) e^{t^{\prime} \partial_{t} G-t^{\prime} a} d t^{\prime}+U$,

or

$H=p^{2} \sigma^{2} \hat{\Theta}(H+a)+U$,

where the front speed is now given by

$v(H)=H \sqrt{\frac{\sigma^{2} \hat{\Theta}(H+a)}{H-U}}, \quad \frac{\partial p}{\partial H}=\frac{p}{H}$.

As has been noted previously [26], the Hamilton-Jacobi hyperbolic scaling approach is equivalent to a linear marginal stability analysis $[27,28]$ used to obtain the selected speed of front propagation, where $\partial H / \partial p=H / p$ is the existence condition for a minimum speed. If the coupling between reactions and dispersal is purely nonlinear, as is the case with Eq. (10), the Hamilton-Jacobi approach by virtue of being equivalent to a linear analysis does not incorporate the contribution of this purely nonlinear coupling. Hence, the linear marginal speed of front propagation is uninfluenced by a purely nonlinear coupling term. However, if the kinetic term contains separate linear birth and death terms, the coupling between reactions and dispersal includes a linear part and has a nontrivial effect on the linear marginal speed of front propagation. This is clearly seen in Eq. (19) through the shifting of the Laplace transform of the memory kernel by the linear death rate $a$. A similar shifting has been found in a two-component reaction-transport model for the migration-proliferation dichotomy in the spreading of tumor cells [29].

A propagating front with non-zero speed only exists if $v(H)$ is a monotonically increasing function of $H$. Then the minimum speed is either provided by $v\left(H^{*}\right)$, where $H^{*}$ is the solution of $\partial H / \partial p=H / p$, which is equivalent to

$$
\frac{d}{d H}\left(\frac{H-U}{\hat{\Theta}(H+a)}\right)=\frac{2(H-U)}{H \hat{\Theta}(H+a)},
$$

or the value of $v(H \rightarrow \infty)$. The Laplace transform of the expansion $e^{-a t} \Theta(t)=\Theta(0) e^{-a t}+e^{-a t} \dot{\Theta}(0) t+e^{-a t} \ddot{\Theta}(0) \times$ $t^{2} / 2+\cdots$, where the overdot indicates a temporal derivative, is given by $\hat{\Theta}(H+a)=\Theta(0)(H+a)^{-1}+\dot{\Theta}(0)(H+a)^{-2}+$ $\ddot{\Theta}(0)(H+a)^{-3}+\cdots$. Therefore in the limit $H \rightarrow \infty$, $\hat{\Theta}(H+a) \sim \Theta(0)(H+a)^{-1}$, and $v(H \rightarrow \infty)=\sqrt{\sigma^{2} \Theta(0)}$. Summarizing, we have

$$
v= \begin{cases}H^{*} \sqrt{\frac{\sigma^{2} \hat{\Theta}\left(H^{*}+a\right)}{H^{*}-U}}, & \Theta(0)=0, \\ \min \left[H^{*} \sqrt{\frac{\sigma^{2} \hat{\Theta}\left(H^{*}+a\right)}{H^{*}-U}}, \sqrt{\sigma^{2} \Theta(0)}\right], & \Theta(0) \neq 0 .\end{cases}
$$

\section{Speed of reaction-subdiffusion fronts}

We now examine the effects of the coupling between reactions and dispersal on front propagation for the case of dispersal by anomalous diffusion, specifically subdiffusion. We consider in the following CTRWs with short-range jump length PDFs $\lambda(r)$, e.g., a Gaussian, and long-tailed waiting time PDFs, e.g., a PDF derived from a Mittag-Leffler function for the survival probability, $l(t)=1-\int_{0}^{t} \phi\left(t^{\prime}\right) d t^{\prime}=E_{\alpha}\left(-t^{\alpha}\right)$ with $0<\alpha<1$ [30]. The asymptotic behavior of the waiting time PDF is given by $\phi(t) \sim t^{-(1+\alpha)}$ as $t \rightarrow \infty$. To take the long-time limit, we consider the scaled waiting time PDF [30]:

$\phi(t) \rightarrow \frac{\phi(t / \eta)}{\eta}, \quad \eta>0$,

which results in

$\hat{\phi}(u) \rightarrow 1-(u \eta)^{\alpha}+o\left(\eta^{\alpha}\right)$.

Consequently, the long-time limit corresponds to setting $\hat{\phi}(u)=$ $1-(u \eta)^{\alpha}$ with $\eta \rightarrow 0$.

With this choice of the waiting time PDF, the memory kernel $\hat{\Theta}(H)$, defined by Eq. (6), takes the form, $\hat{\Theta}(H)=[H \eta]^{1-\alpha} / \eta$. Thus,

$\hat{\Theta}(H+a)=[(H+a) \eta]^{1-\alpha} / \eta$.

To evaluate the propagation speed, we solve Eq. (20) with the memory kernel given by Eq. (24). The physically acceptable solution of the resulting quadratic equation is

$H^{*}=\frac{a+U(\alpha-3)-W}{2(\alpha-2)}$,

where

$W=\sqrt{8 a U(2-\alpha)+(-a+3 U-U \alpha)^{2}}$,

and the front speed is obtained by substituting $H^{*}$ into Eq. (21). The second solution of the quadratic equation is rejected on physical grounds, since it leads to negative front speeds. Moreover, since $\Theta(0)$ does not exist, the selected front speed is

$v=H^{*} \sqrt{\sigma^{2} \hat{\Theta}\left(H^{*}+a\right) /\left(H^{*}-U\right)}$.

As expected, choosing $\alpha=1$ and defining as usual the diffusion coefficient $D=\sigma^{2} / \eta$, we recover $v=2 \sqrt{\sigma^{2} U / \eta}=$ $2 \sqrt{D U}$, the speed of front propagation for the case of classical diffusion. Eq. (27) is derived from the generalized reactiondiffusion equation (5) and is valid in the "diffusive" regime, which is attained if the chemical times $1 / U, 1 / a$ and $1 / b$ are large compared to the transport time scale $\eta$, i.e., $U \eta \sim a \eta \sim b \eta$ are small. This condition is well known from the study of hyperbolic reaction-diffusion systems [5,31]. It simply reflects the fact that "diffusive" behavior results from the accumulation of many jumps of the random walk on a time scale much larger than $\eta$. Therefore large reaction rates $U$ require corresponding small values of $\eta$. For subdiffusive transport, the condition that $U \eta \sim a \eta \sim b \eta$ are small ensures that the speed is a monotonically increasing function of $\alpha$. This is the correct physical behavior because the transport is faster as $\alpha$ tends to 1 and slower as $\alpha$ tends to 0 . A typical plot of this behavior is shown in Fig. 1. As illustrated by Fig. 1, choosing $a=0$ always results in the underestimation of the propagation speed.

It is important to note that the propagation speed for reaction-subdiffusion fronts may be rewritten in terms of a gen- 


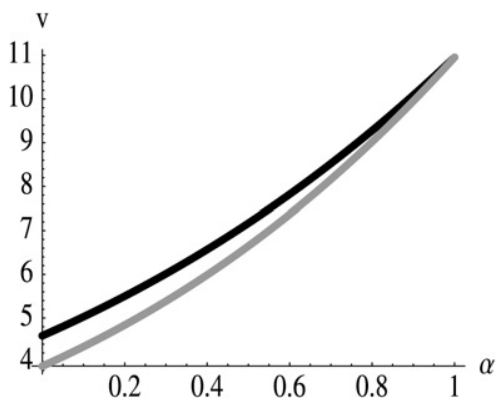

Fig. 1. The gray curve is a plot of $v$ as a function of $\alpha$ when $a=0$. The black curve is a plot of $v$ vs. $\alpha$ for $a=0.9$. The values of the other parameters are: $U=1.5, \sigma=1.0$, and $\eta=0.05$.

eralized diffusion coefficient $K_{\alpha} \equiv \sigma^{2} / \eta^{\alpha}[32]$,

$v=H^{*} \sqrt{K_{\alpha}} \sqrt{\frac{\left(H^{*}+a\right)^{1-\alpha}}{\left(H^{*}-U\right)}}$.

This is of significance in experimental contexts where only the value of the subdiffusion coefficient $K_{\alpha}$, and not the underlying CTRW time scale $\eta$, is measurable [33].

\section{Conclusions}

The memory inherent in non-standard diffusive transport induces a coupling between reaction and dispersal processes. We have suggested a generalization of Fick's first law for reactiontransport systems with non-standard diffusion that accounts for this coupling. The effect of memory on front propagation is investigated by carrying out a hyperbolic scaling for the generalized reaction-diffusion equation with FKPP-type kinetics. Besides deriving a general, analytical expression for the minimal speed, we focus in particular on the case where the dispersal is governed by anomalous diffusion, namely subdiffusion. For FKPP-type kinetics, the front speed is determined by the linear marginal stability criterion, and the coupling between transport and reactions only plays a role for kinetic schemes with linear death rates. Neglecting the coupling in the latter case results in an underestimation of the front propagation speed for subdiffusive transport. We conclude by mentioning an open problem that deserves further attention. The effect of coupling between reactions and dispersal needs to be explored for propagating fronts where the linear marginal stability analysis is not applicable. We expect the coupling to effect the speed of such fronts even if the death rate is nonlinear.

\section{Acknowledgement}

This work has been supported by the National Science Foundation under Grant No. CHE-0533642 (W.H.) and the MICYT under grants FIS2006-12296-C02-01 and SGR200500087 (V.M.) and EPSRC EP/D03115X/1.

\section{References}

[1] M.C. Cross, P.C. Hohenberg, Rev. Mod. Phys. 65 (1993) 851.

[2] J.D. Murray, Mathematical Biology II: Spatial Models and Biomedical Applications, Springer, New York, 2003.

[3] R.J. Field, M. Burger (Eds.), Oscillations and Traveling Waves in Chemical Systems, Wiley, New York, 1985.

[4] P.C. Fife, Mathematical Aspects of Reacting and Diffusing Systems, Lecture Notes in Biomathematics, vol. 28, Springer-Verlag, Berlin, 1979.

[5] W. Horsthemke, Phys. Lett. A 263 (1999) 285.

[6] J. Fort, V. Méndez, Phys. Rev. Lett. 82 (1999) 867.

[7] J. Fort, V. Méndez, Phys. Rev. Lett. 89 (2002) 178101.

[8] V. Ortega-Cejas, J. Fort, V. Méndez, Ecology 85 (2004) 258.

[9] K.K. Manne, A.J. Hurd, V.M. Kenkre, Phys. Rev. E 61 (2000) 4177.

[10] G. Abramson, A.R. Bishop, V.M. Kenkre, Phys. Rev. E 64 (2001) 066615.

[11] S. Fedotov, V. Méndez, Phys. Rev. E 66 (2002) 030102.

[12] D. del-Castillo-Negrete, B.A. Carreras, V.E. Lynch, Phys. Rev. Lett. 91 (2002) 018302.

[13] S. Fedotov, Y. Okuda, Phys. Rev. E 66 (2002) 021113.

[14] V. Méndez, V. Ortega-Cejas, J. Casas-Vázquez, Eur. Phys. J. 53 (2006) 503.

[15] V. Méndez, S. Fedotov, W. Horsthemke, Europhys. Lett. 77 (2007) 58006.

[16] D.D. Joseph, L. Preziosi, Rev. Mod. Phys. 61 (1989) 41.

[17] A. Yadav, W. Horsthemke, Phys. Rev. E 74 (2006) 066118.

[18] M.O. Vlad, J. Ross, Phys. Rev. E 66 (2002) 061908.

[19] H.P. Breuer, W. Huber, F. Petruccione, Physica D 73 (1994) 259.

[20] J. Mai, I.M. Sokolov, A. Blumen, Phys. Rev. Lett. 77 (1996) 4462.

[21] E. Brunet, B. Derrida, Phys. Rev. E 56 (1997) 2597.

[22] D.A. Kessler, Z. Ner, L.M. Sanders, Phys. Rev. E 58 (1998) 107.

[23] T. Callaghan, E. Khain, L.M. Sander, R.M. Ziff, J. Stat. Phys. 122 (2006) 909.

[24] R. Fisher, Ann. Eugen. 7 (1937) 355; A.N. Kolmogorov, I.G. Petrovskii, N.S. Piskunov, Univ. Bull. Math. Ser. A 1 (1937) 1.

[25] F. Schlögl, Z. Phys. 253 (1972) 147.

[26] V. Méndez, D. Campos, S. Fedotov, Phys. Rev. E 70 (2004) 036121.

[27] W. van Saarloos, Phys. Rev. A 39 (1989) 6367.

[28] W. van Saarlos, Phys. Rep. 386 (2003) 29.

[29] S. Fedotov, A. Iomin, Phys. Rev. Lett. 98 (2007) 118101.

[30] E. Scalas, R. Gorenflo, F. Mainardi, Phys. Rev. E 69 (2004) 011107.

[31] S. Fedotov, Phys. Rev. E 58 (1998) 5143.

[32] R. Metzler, J. Klafter, Phys. Rep. 339 (2000) 1.

[33] T. Koztołowicz, K. Dworecki, St. Mrówczyński, Phys. Rev. E 71 (2005) 041105. 